\title{
Statistical analysis with cosmic-expansion-rate measurements and two-point diagnostics
}

\author{
Xiaogang Zheng ${ }^{1,2,3}$, Marek Biesiada ${ }^{2,3}$, Xuheng Ding ${ }^{1,2}$, Shuo Cao ${ }^{2, a}$, Sixuan Zhang ${ }^{2}$, Zong-Hong Zhu ${ }^{1,2}$ \\ ${ }^{1}$ School of Physics and Technology, Wuhan University, Wuhan 430072, China \\ ${ }^{2}$ Department of Astronomy, Beijing Normal University, Beijing 100875, China \\ ${ }^{3}$ Department of Astrophysics and Cosmology, Institute of Physics, University of Silesia, Uniwersyecka 4, 40-007 Katowice, Poland
}

Received: 16 January 2018 / Accepted: 22 March 2018 / Published online: 29 March 2018

(C) The Author(s) 2018

\begin{abstract}
Direct measurements of Hubble parameters $H(z)$ are very useful for cosmological model parameters inference. Based on them, Sahni, Shafieloo and Starobinski introduced a two-point diagnostic $\mathrm{Omh}^{2}\left(z_{i}, z_{j}\right)$ as an interesting tool for testing the validity of the $\Lambda \mathrm{CDM}$ model. Applying this test they found a tension between observations and predictions of the $\Lambda \mathrm{CDM}$ model. We use the most comprehensive compilation $H(z)$ data from baryon acoustic oscillations (BAO) and differential ages (DA) of passively evolving galaxies to study cosmological models using the Hubble parameters itself and to distinguish whether $\Lambda \mathrm{CDM}$ model is consistent with the observational data with statistical analysis of the corresponding $\operatorname{Omh}^{2}\left(z_{i}, z_{j}\right)$ two-point diagnostics. Our results show that presently available $H(z)$ data significantly improve the constraints on cosmological parameters. The corresponding statistical $O m h^{2}\left(z_{i}, z_{j}\right)$ two-point diagnostics seems to prefer the quintessence with $w>-1$ over the $\Lambda$ CDM model. Better and more accurate prior knowledge of the Hubble constant, will considerably improve the performance of the statistical $O m h^{2}\left(z_{i}, z_{j}\right)$ method.
\end{abstract}

\section{Introduction}

The discovery of accelerating expansion of the Universe $[1,2]$ created a big challenge for the modern science and stimulated cosmologists to investigate the essentials of this phenomenon. In order to explain present acceleration of the Universe, there should exist some mechanism providing a repulsive effect. There are two broad ways of achieving this: considering the modified gravity [3] or adding an exotic dark energy component [4] to the matter content of the Universe. The simplest solution along the second line of reasoning is the $\Lambda \mathrm{CDM}$ model in which the cosmological constant $\Lambda$ acts

a e-mail: caoshuo@bnu.edu.cn as a repulsive component in addition to ordinary cold dark matter and - now dynamically unimportant - CMB radiation or cosmic neutrinos. However, the cosmological constant, while being the most parsimonious choice is far from being a satisfactory explanation both theoretically (fine tuning and coincidence problems) and from the observational point of view [5]. Because there is no clear theoretical preference for the alternative to the $\Lambda \mathrm{CDM}$ model, it is reasonable to take a phenomenological approach to parameterize the unknown by hypothetical fluid with an equation of state $p=w \rho$ where $w$ coefficient might be constant or allowed to vary with cosmic time $w(z)=w_{0}+w_{a} \frac{z}{1+z}[6,7]$. Such models are known as $\mathrm{wCDM}$ and CPL, respectively. Standard $\Lambda \mathrm{CDM}$ is nested within such classes of models.

The most straightforward technique to constrain cosmological equation of state is by constructing the Hubble diagram $d_{L, A}(z)$ using either luminosity or angular diameter distances to the objects whose redshifts are known [8-12]. This approach demands either standard candles like SN Ia or standard rulers like CMB acoustic peaks or BAO. One should be cautious, however, about the way they are calibrated in order not to fall into circularity problems with respect to the cosmological model assumed during the calibration. From this perspective, another very attractive probe - Hubble function at different redshifts $H(z)-$ is becoming accessible. In particular, $H(z)$ measurements from the so called cosmic chronometers, i.e. differential ages (DA) of passively evolving galaxies are free from any prior assumption concerning cosmology, only uncertainty being of astrophysical origin (the adopted population synthesis model).

Recently, using DA technique, Moresco et al. [13,14] provided another few $H(z)$ measurements in addition to already existing data (see Ding et al. [15] for the compilation). They also used the whole compilation of $H(z)$ from DA to constrain cosmology [16]. Expansion rates at different redshifts not only allowed to use this pure information for cosmo- 
graphic purposes but opened also a new chapter in using the so called $O m(z)$ diagnostics. They were introduced by [17] in order to distinguish between $\triangle \mathrm{CDM}$ and other dark energy scenarios. This diagnostics is defined as

$O m(z) \equiv \frac{E^{2}(z)-1}{(1+z)^{3}-1}$

where $E(z) \equiv H(z) / H_{0}$ is the dimensionless expansion rate and in the $\Lambda C D M$ model it should be equal exactly to the present value of matter density $\operatorname{Om}(z)=\Omega_{m, 0}$. Its advantage as a screening test for the $\Lambda \mathrm{CDM}$ (formal function of the redshift $O m(z)$ should be just a constant) is clear. Later on, they developed it further by introducing a two-point diag$\operatorname{nostic} O m h^{2}\left(z_{i}, z_{j}\right)[18]$

$O m h^{2}\left(z_{i}, z_{j}\right)=\frac{h^{2}\left(z_{i}\right)-h^{2}\left(z_{j}\right)}{\left(1+z_{i}\right)^{3}-\left(1+z_{j}\right)^{3}}$

where $h(z) \equiv H(z) / 100$, and subsequently used it in [19] to perform this test on three accurately measured values of $H(z)$ from BAO demonstrating a tension with the value of $\Omega_{m, 0} h^{2}$ given by Ade et al. [20]. Later, Ding et al. [15] collected a larger $H(z)$ sample (6 from BAO measurements and 23 from DA measurements) to do this test confirming that the tension exists. The two-point diagnostics has an advantage that if we know Hubble parameters at $n$ different redshifts, we can get $n(n-1) / 2$ pairs of data. This enlargement of statistical sample for inference occurs at the expense of nontrivial statistical properties of observables [21].

As already mentioned, $H(z)$ can be used as a cosmological probe to constrain cosmological parameters directly [22-25]. However, it is also tempting to perform the fit cosmological parameters based on the two-point diagnostics. Therefore, in this paper we constrain the cosmological models not only using $H(z)$ directly, but also using the two-point $O m h^{2}\left(z_{i}, z_{j}\right)$ diagnostic. The rest of the paper is organized as follows. In Sect. 2, we briefly introduce the observational Hubble parameters, and present our methodology to constrain cosmology with $O m h^{2}\left(z_{i}, z_{j}\right)$ probe. We show our results followed by discussion in Sect. 3. Finally, we conclude in Sect. 4.

\section{Data and method}

2.1 Empirical $H(z)$ data and constraints based directly on them

We used a collection of totally 36 measurements of $H(z)$ shown in Fig. 1. Among them, 30 data points come from cosmic chronometers [13,14,26-30], i.e. the differential ages of passively evolving galaxies as a function of redshift. Other

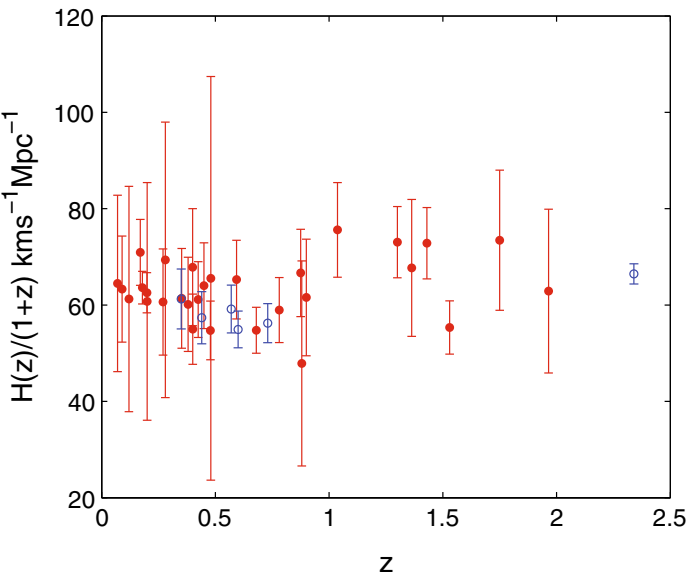

Fig. 1 Observed $H(z)$ data from DA (red dot) and BAO (blue circle) with corresponding uncertainties

6 points come from the BAO peak position as a standard ruler in the radial direction [31-34]. Because the $H(z)$ data come from two different techniques, and moreover one of the BAO points - the one at the highest redshift [34] - was obtained in a different way than other BAO data (from the $L y \alpha$ forest) we also divided our data set (full $n=36$ sample) into subsamples: $n=35$ points - high $z$ BAO excluded, $n=30-$ from cosmic chronometers (DA) only and $n=6$ from BAO only. Such division is dictated by desire to reveal possible systematics due to inhomogeneous sample.

We will use these data to estimate cosmological parameters denoted in short as $\mathbf{p}$. In particular, $\mathbf{p}=\left\{\Omega_{m, 0}, w\right\}$ for $\mathrm{wCDM}$ and $\mathbf{p}=\left\{\Omega_{m, 0}, w_{0}, w_{a}\right\}$ for CPL model. It is obvious that $\Lambda \mathrm{CDM}$ model with $\mathbf{p}=\left\{\Omega_{m, 0}\right\}$ is nested within the above mentioned models and is equivalent to WCDM with $w$ parameter fixed at $w=-1$ or CPL with $w_{0}=-1$ and $w_{a}=0$ fixed, so in this case $\mathbf{p}=\Omega_{m, 0}$. For completeness and cross-checks we will also report fits on the present matter density parameter in $\Lambda \mathrm{CDM}$ model. Let us note that we do not consider the Hubble constant $H_{0}$ as a free parameter for fitting. Therefore, as described in details below, we either marginalize over $H_{0}$ (in some specific way) or use an informative prior for it. In order to estimate the best fitted values of these parameters we will maximize the likelihood derived from the $\chi^{2}$ function. In the case of constraints based on $H(z)$ data it reads:

$\chi_{H(z)}^{2}\left(H_{0}, \mathbf{p}\right)=\sum_{i=1}^{n}\left[\frac{H\left(z_{i} ; H_{0}, \mathbf{p}\right)_{t h}-H\left(z_{i}\right)_{o b s}}{\sigma_{H\left(z_{i}\right)_{o b s}}}\right]^{2}$

Because we treat $H_{0}$ as a nuisance parameter, one can factor it out: $H\left(z ; H_{0}, \mathbf{p}\right)_{t h}=H_{0} E(z ; \mathbf{p})$ and rewrite Eq. (3) in the following way 


$$
\begin{aligned}
\chi_{H(z)}^{2}\left(H_{0}, \mathbf{p}\right)= & \sum_{i=1}^{n}\left[\frac{H_{0} E\left(z_{i} ; \mathbf{p}\right)-H\left(z_{i}\right)_{o b s}}{\sigma_{H\left(z_{i}\right)_{o b s}}}\right]^{2} \\
= & H_{0}^{2} \sum_{i=1}^{n} \frac{E^{2}\left(z_{i} ; \mathbf{p}\right)}{\sigma_{H\left(z_{i}\right)_{o b s}}^{2}}+\sum_{i=1}^{n} \frac{H\left(z_{i}\right)_{o b s}^{2}}{\sigma_{H\left(z_{i}\right)_{o b s}}^{2}} \\
& -2 H_{0} \sum_{i=1}^{n} \frac{H\left(z_{i}\right)_{o b s} E\left(z_{i} ; \mathbf{p}\right)}{\sigma_{H\left(z_{i}\right)_{o b s}}^{2}}
\end{aligned}
$$

where only the dimensionless expansion rate depends explicitly on the cosmological model parameters. Let us recall that in the wCDM model with constant $w$ coefficient in the equation of state it reads:

$$
\begin{aligned}
& E\left(z ; w, \Omega_{m, 0}\right) \\
& \quad=\left(\Omega_{m, 0}(1+z)^{3}+\left(1-\Omega_{m, 0}\right)(1+z)^{3(1+w)}\right)^{1 / 2}
\end{aligned}
$$

while and for the Chevalier-Polarski-Linder (CPL) parametrization $[6,7]$, one has:

$$
\begin{aligned}
& E\left(z ; w, \Omega_{m, 0}\right) \\
& =\left(\Omega_{m, 0}(1+z)^{3}+\left(1-\Omega_{m, 0}\right)(1+z)^{3\left(1+w_{0}+w_{a}\right)} e^{\left(-\frac{3 w_{a} z}{1+z}\right)}\right)^{1 / 2}
\end{aligned}
$$

Introducing auxiliary quantities:

$$
\begin{aligned}
Q_{1} & =\sum_{i=1}^{n} \frac{E^{2}\left(z_{i} ; \mathbf{p}\right)}{\sigma_{H\left(z_{i}\right)_{o b s}}^{2}} \\
Q_{2} & =\sum_{i=1}^{n} \frac{H_{o b s}\left(z_{i}\right) E\left(z_{i} ; \mathbf{p}\right)}{\sigma_{H\left(z_{i}\right)_{o b s}}^{2}} \\
Q_{3} & =\sum_{i=1}^{n} \frac{H_{o b s}^{2}\left(z_{i}\right)}{\sigma_{H\left(z_{i}\right)_{o b s}}^{2}}
\end{aligned}
$$

one can rewrite Eq. (4) as

$$
\chi_{H(z)}^{2}\left(H_{0}, \mathbf{p}\right)=Q_{1} H_{0}^{2}-2 Q_{2} H_{0}+Q_{3}
$$

Now, it is easy to see that the reduced Chi-square minimized with respect to the nuisance parameter $H_{0}$ is equal to

$\chi_{H(z)}^{2}(\mathbf{p})=Q_{3}-\frac{Q_{2}^{2}}{Q_{1}}$

at $H_{0}=Q_{2} / Q_{1}$ and one can use it further to constrain parameters $\mathbf{p}$ without any prior assumptions about $H_{0}$. This approach is alternative to standard procedure of marginalizing over $H_{0}$.

Another approach is to take an informative prior for $H_{0}$. Following Farooq [35], we will assume that the prior distribution of $H_{0}$ is Gaussian with the mean $\bar{H}_{0}$ and the standard deviation $\sigma_{H_{0}}$ :
$P\left(H_{0}\right)=\frac{1}{\sqrt{2 \pi \sigma_{H_{0}}^{2}}} e^{-\left(H_{0}-\bar{H}_{0}\right)^{2} /\left(2 \sigma_{H_{0}}^{2}\right)}$

Then, we can build the posterior likelihood function $\mathcal{L}_{H}(\mathbf{p})$ by marginalizing over $H_{0}$

$\mathcal{L}_{H}(\mathbf{p})=\int_{0}^{\infty} e^{-\chi_{H(z)}^{2}\left(H_{0}, \mathbf{p}\right)} P\left(H_{0}\right) d H_{0}$

Introducing

$\alpha=\frac{1}{\sigma_{H_{0}}^{2}}+Q_{1}$

$\beta=\frac{\bar{H}_{0}}{\sigma_{H_{0}}^{2}}+Q_{2}$

$\gamma=\frac{\bar{H}_{0}^{2}}{\sigma_{H_{0}}^{2}}+Q_{3}$

where the terms $Q_{1}, Q_{2}, Q_{3}$ are the same as in Eq. (7), and performing the integral analytically one arrives at the following expression for the posterior likelihood:

$\mathcal{L}_{H}(\mathbf{p})=\frac{1}{2 \sqrt{\alpha \sigma_{H_{0}}^{2}}} e^{\left[-\frac{1}{2}\left(\gamma-\frac{\beta^{2}}{\alpha}\right)\right]}\left[1+\operatorname{erf}\left(\frac{\beta}{\sqrt{2 \alpha}}\right)\right]$

where $\operatorname{erf}(x)=\frac{2}{\sqrt{\pi}} \int_{0}^{x} e^{-t^{2}} d t$. Details of the derivation can be found in Farooq [35].

Then, we maximize the likelihood $\mathcal{L}_{H}(\mathbf{p})$, with respect to the parameters $\mathbf{p}$ in order to find the best-fitted parameter values $\mathbf{p}_{0}$.

\subsection{Constraints based on two-point diagnostics}

So far, the two point diagnostic has been mostly used to test the validity of $\Lambda \mathrm{CDM}$ model and to some extent its generalizations $[15,19,21]$. Here, we will use the $O m h^{2}\left(z_{i}, z_{j}\right)$ function for the purpose of constraining cosmological parameters $\mathbf{p}$ following the similar strategy as described above for expansion rates alone.

Introducing the simplifying notation: $h(z)=H(z) / 100$ and $e(z)=E(z) / 100$, one can express theoretically expected $O m h^{2}\left(z_{i}, z_{j} ; H_{0}, \mathbf{p}\right)_{t h}$ and observed $O m h^{2}\left(z_{i}, z_{j}\right)_{o b s}$ two point diagnostics as

$$
\begin{aligned}
O m h^{2}\left(z_{i}, z_{j} ; H_{0}, \mathbf{p}\right)_{t h} & =\frac{\left[H_{0} e\left(z_{i}\right)\right]^{2}-\left[H_{0} e\left(z_{j}\right)\right]^{2}}{\left(1+z_{i}\right)^{3}-\left(1+z_{j}\right)^{3}} \\
O m h^{2}\left(z_{i}, z_{j}\right)_{o b s} & =\frac{\left[h\left(z_{i}\right)\right]^{2}-\left[h\left(z_{j}\right)\right]^{2}}{\left(1+z_{i}\right)^{3}-\left(1+z_{j}\right)^{3}}
\end{aligned}
$$

The $\chi^{2}$ function for the $O m h^{2}\left(z_{i}, z_{j}\right)$ two point diagnostics is 
Table 1 Best fitted parameters in $\Lambda \mathrm{CDM}$ cosmological model using $H(z)$ data alone and $\operatorname{Omh}^{2}\left(z_{i}, z_{j}\right)$ two-point diagnostics. Fits done on different sub-samples are reported. First part corresponds to the reduced $\chi^{2}$ method. From second to fifth part corresponds to the other two different methods priors on $H_{0}$ taken after Planck [20] and after [37]

\begin{tabular}{|c|c|c|}
\hline & $\Omega_{m, 0}$ & $\chi_{\text {d.o.f }}^{2}$ \\
\hline \multicolumn{3}{|l|}{$\chi_{H(z)}^{2}(\mathbf{p})$ reduced Chi-square } \\
\hline$n=6 \mathrm{BAO} \mathrm{H}(\mathrm{z})$ & $0.28_{-0.07}^{+0.09}$ & $0.64 / 5$ \\
\hline$n=30 \mathrm{DA} \mathrm{H}(\mathrm{z})$ & $0.32_{-0.08}^{+0.10}$ & $14.50 / 29$ \\
\hline$n=35 \mathrm{H}(\mathrm{z})$ & $0.31_{-0.08}^{+0.09}$ & $16.84 / 34$ \\
\hline$n=36$ all $\mathrm{H}(\mathrm{z})$ & $0.26_{-0.04}^{+0.05}$ & $17.78 / 35$ \\
\hline $\mathcal{L}_{H}(\mathbf{p})$ Gaussian prior & \multicolumn{2}{|l|}{$H_{0}=67.4 \pm 1.4 k m s^{-1} M p c^{-1}$} \\
\hline$n=6 \mathrm{BAO} \mathrm{H}(\mathrm{z})$ & $0.270_{-0.032}^{+0.034}$ & $1.22 / 5$ \\
\hline$n=30 \mathrm{DA} \mathrm{H}(\mathrm{z})$ & $0.329_{-0.053}^{+0.062}$ & $15.14 / 29$ \\
\hline$n=35 \mathrm{H}(\mathrm{z})$ & $0.310_{-0.048}^{+0.051}$ & $16.62 / 34$ \\
\hline$n=36$ all $\mathrm{H}(\mathrm{z})$ & $0.281_{-0.031}^{+0.032}$ & $19.17 / 35$ \\
\hline $\mathcal{L}_{H}(\mathbf{p})$ Gaussian prior & \multicolumn{2}{|l|}{$H_{0}=73.24 \pm 1.74 \mathrm{~km} \mathrm{~s}^{-1} M p c^{-1}$} \\
\hline$n=6 \mathrm{BAO} \mathrm{H}(\mathrm{z})$ & $0.224_{-0.029}^{+0.032}$ & $2.80 / 5$ \\
\hline$n=30 \mathrm{DA} \mathrm{H}(\mathrm{z})$ & $0.261_{-0.047}^{+0.056}$ & $17.38 / 29$ \\
\hline$n=35 \mathrm{H}(\mathrm{z})$ & $0.241_{-0.042}^{+0.050}$ & $20.45 / 34$ \\
\hline$n=36$ all $\mathrm{H}(\mathrm{z})$ & $0.238_{-0.028}^{+0.031}$ & $20.70 / 35$ \\
\hline$\chi_{O m h^{2}}^{2}\left(H_{0}, \mathbf{p}\right)$ Uniform prior & \multicolumn{2}{|l|}{$H_{0} \in[66.0,68.8] \mathrm{km} \mathrm{s}^{-1} \mathrm{Mpc}^{-1}$} \\
\hline$n=6 \mathrm{BAO} \mathrm{H}(\mathrm{z})$ & $0.272_{-0.017}^{+0.018}$ & $1.62 / 13$ \\
\hline$n=30 \mathrm{DA} \mathrm{H}(\mathrm{z})$ & $0.318_{-0.019}^{+0.019}$ & $220.46 / 433$ \\
\hline$n=35 \mathrm{H}(\mathrm{z})$ & $0.311_{-0.016}^{+0.017}$ & $296.21 / 593$ \\
\hline$n=36$ all $\mathrm{H}(\mathrm{z})$ & $0.279_{-0.011}^{+0.011}$ & $323.33 / 628$ \\
\hline$\chi_{O m h^{2}}^{2}\left(H_{0}, \mathbf{p}\right)$ Uniform prior & \multicolumn{2}{|l|}{$H_{0} \in[71.50,74.98] \mathrm{km} \mathrm{s}^{-1} \mathrm{Mpc}^{-1}$} \\
\hline$n=6 \mathrm{BAO} \mathrm{H}(\mathrm{z})$ & $0.229_{-0.015}^{+0.016}$ & $1.62 / 13$ \\
\hline$n=30 \mathrm{DA} \mathrm{H}(\mathrm{z})$ & $0.268_{-0.017}^{+0.017}$ & $220.46 / 433$ \\
\hline$n=35 \mathrm{H}(\mathrm{z})$ & $0.261_{-0.015}^{+0.016}$ & $296.21 / 593$ \\
\hline$n=36$ all $\mathrm{H}(\mathrm{z})$ & $0.234_{-0.011}^{+0.012}$ & $323.33 / 628$ \\
\hline
\end{tabular}

$$
\begin{aligned}
& \chi_{O m h^{2}}^{2}\left(H_{0}, \mathbf{p}\right) \\
& =\sum_{i=1}^{n-1} \sum_{j=i+1}^{n}\left[\frac{O m h^{2}\left(z_{i}, z_{j} ; H_{0}, \mathbf{p}\right)_{t h}-O m h^{2}\left(z_{i}, z_{j}\right)_{o b s}}{\sigma_{O m h^{2}\left(z_{i}, z_{j}\right)_{o b s}}}\right]^{2}
\end{aligned}
$$

Then, we minimize this $\chi^{2}$ function to find the best-fitted cosmological parameters.

\section{Results and discussion}

Let us discuss the results starting with $\Lambda \mathrm{CDM}$ model. Numerical details are displayed in Table 1 and comprise fits of $\Omega_{m, 0}$ on different sub-samples using three techniques: reduced Chi-square Eq. (9), Chi-square with Gaussian priors on $H_{0}$ and $O m h^{2}$ two-point diagnostics. Full data-set without the high- $z$ BAO point (i.e. $n=35$ data points), gives $\Omega_{m, 0}=$ $0.30_{-0.07}^{+0.10}$ and $H_{0}=67.55_{-4.33}^{+4.57} \mathrm{~km} \mathrm{~s}^{-1} \mathrm{Mpc}^{-1}$, whereas homogeneous DA sample ( $n=30$ data points) results with
$\Omega_{m, 0}=0.32_{-0.08}^{+0.10}$ and $H_{0}=67.74_{-4.37}^{+4.95} \mathrm{~km} \mathrm{~s}^{-1} \mathrm{Mpc}^{-1}$, respectively. These two central values are very close to Planck central fits result [20], that is $\Omega_{m, 0}=0.314 \pm 0.020$ and $H_{0}=67.4 \pm 1.4 \mathrm{~km} \mathrm{~s}^{-1} \mathrm{Mpc}^{-1}$. When the high$z$ point included in the sub-sample, the results changes to $\Omega_{m, 0}=0.28_{-0.07}^{+0.09}$ and $\Omega_{m, 0}=0.26_{-0.04}^{+0.05}$ for the $6 \mathrm{H}(\mathrm{z})$ from $\mathrm{BAO}$ and the whole $\mathrm{H}(\mathrm{z})$ sample, respectively. And corresponding Hubble constant are $H_{0}=66.04_{-6.86}^{+7.89}$ and $H_{0}=69.14_{-3.54}^{+3.75} \mathrm{~km} \mathrm{~s}^{-1} M p c^{-1}$, respectively. The result is still consistent with Planck result in $1 \sigma$ but there is a mismatch in central fits. Results obtained with priors on $H_{0}$ reveal that $\Omega_{m, 0}$ fit is sensitive to the Hubble constant assumed. Inclusion of the $H(z=2.34)$ point leads to results which are biased with respect to Planck result. One can also see that $O m h^{2}\left(z_{i}, z_{j}\right)$ two-point diagnostics gives more stringent results than $H(z)$ alone.

Results concerning wCDM model are reported in Table 2 and shown on Figs. 2 and 3. When we take the prior $H_{0}=67.4 \pm 2.4 \mathrm{~km} \mathrm{~s}^{-1} \mathrm{Mpc}^{-1}$ from [20], the dark energy equation of state constraint is almost totally consistent with 
Table 2 Best fitted parameters in wCDM cosmological model using $H(z)$ data alone and $O m h^{2}\left(z_{i}, z_{j}\right)$ two-point diagnostics. Fits done on different sub-samples are reported. First part corresponds to the reduced $\chi^{2}$ method. From second to fifth part corresponds to the other two different methods priors on $H_{0}$ taken after Planck [20] and after [37]

\begin{tabular}{|c|c|c|c|}
\hline & $\Omega_{m, 0}$ & $w$ & $\chi_{\text {d.o.f }}^{2}$ \\
\hline \multicolumn{4}{|l|}{$\chi_{H(z)}^{2}(\mathbf{p})$ reduced Chi-square } \\
\hline$n=6 \mathrm{BAO} \mathrm{H}(\mathrm{z})$ & $0.26_{-0.18}^{+0.15}$ & $-0.37_{-2.07}^{+0.11}$ & $0.48 / 4$ \\
\hline$n=30 \mathrm{DA} \mathrm{H}(\mathrm{z})$ & $0.29_{-0.09}^{+0.13}$ & $-1.21_{-1.40}^{+0.69}$ & $14.36 / 28$ \\
\hline$n=35 \mathrm{H}(\mathrm{z})$ & $0.28_{-0.05}^{+0.10}$ & $-1.53_{-1.06}^{+0.70}$ & $15.77 / 33$ \\
\hline$n=36$ all $\mathrm{H}(\mathrm{z})$ & $0.26_{-0.04}^{+0.06}$ & $-0.95_{-0.49}^{+0.40}$ & $17.76 / 34$ \\
\hline $\mathcal{L}_{H}(\mathbf{p})$ Gaussian prior & \multicolumn{3}{|l|}{$H_{0}=67.4 \pm 1.4 k m s^{-1} M p c^{-1}$} \\
\hline$n=6 \mathrm{BAO} \mathrm{H}(\mathrm{z})$ & $0.28_{-0.05}^{+0.04}$ & $-1.04_{-0.34}^{+0.28}$ & $1.19 / 4$ \\
\hline$n=30 \mathrm{DA} \mathrm{H}(\mathrm{z})$ & $0.34_{-0.13}^{+0.08}$ & $-0.97_{-0.55}^{+0.36}$ & $15.14 / 28$ \\
\hline$n=35 \mathrm{H}(\mathrm{z})$ & $0.34_{-0.11}^{+0.07}$ & $-1.08_{-0.49}^{+0.31}$ & $17.43 / 33$ \\
\hline$n=36$ all $\mathrm{H}(\mathrm{z})$ & $0.27_{-0.05}^{+0.04}$ & $-0.90_{-0.25}^{+0.20}$ & $18.74 / 34$ \\
\hline $\mathcal{L}_{H}(\mathbf{p})$ Gaussian prior & \multicolumn{3}{|l|}{$H_{0}=73.24 \pm 1.74 \mathrm{~km} \mathrm{~s}^{-1} M p c^{-1}$} \\
\hline$n=6 \mathrm{BAO} \mathrm{H}(\mathrm{z})$ & $0.24_{-0.04}^{+0.03}$ & $-1.18_{-0.38}^{+0.24}$ & $1.20 / 4$ \\
\hline$n=30 \mathrm{DA} \mathrm{H}(\mathrm{z})$ & $0.32_{-0.09}^{+0.05}$ & $-1.40_{-0.56}^{+0.45}$ & $15.16 / 28$ \\
\hline$n=35 \mathrm{H}(\mathrm{z})$ & $0.31_{-0.07}^{+0.05}$ & $-1.45_{-0.53}^{+0.41}$ & $16.70 / 33$ \\
\hline$n=36$ all $\mathrm{H}(\mathrm{z})$ & $0.25_{-0.03}^{+0.03}$ & $-1.15_{-0.25}^{+0.22}$ & $19.25 / 34$ \\
\hline$\chi_{O m h^{2}}^{2}\left(H_{0}, \mathbf{p}\right)$ Uniform prior & \multicolumn{3}{|l|}{$H_{0} \in[66.0,68.8] \mathrm{km} \mathrm{s}^{-1} M p c^{-1}$} \\
\hline$n=6 \mathrm{BAO} \mathrm{H}(\mathrm{z})$ & $0.28_{-0.03}^{+0.01}$ & $<-0.82$ & $1.18 / 12$ \\
\hline$n=30 \mathrm{DA} \mathrm{H}(\mathrm{z})$ & $0.30_{-0.10}^{+0.03}$ & $-0.80_{-0.32}^{+0.22}$ & $217.08 / 432$ \\
\hline$n=35 \mathrm{H}(\mathrm{z})$ & $0.33_{-0.03}^{+0.02}$ & $-1.20_{-0.40}^{+0.26}$ & $292.35 / 592$ \\
\hline$n=36$ all $\mathrm{H}(\mathrm{z})$ & $0.25_{-0.02}^{+0.02}$ & $-0.78_{-0.11}^{+0.11}$ & $312.82 / 627$ \\
\hline$\chi_{O m h^{2}}^{2}\left(H_{0}, \mathbf{p}\right)$ Uniform prior & \multicolumn{3}{|l|}{$H_{0} \in[71.50,74.98] \mathrm{km} \mathrm{s}^{-1} M p c^{-1}$} \\
\hline$n=6 \mathrm{BAO} \mathrm{H}(\mathrm{z})$ & $0.24_{-0.02}^{+0.01}$ & $<-0.88$ & $1.16 / 12$ \\
\hline$n=30 \mathrm{DA} \mathrm{H}(\mathrm{z})$ & $0.26_{-0.07}^{+0.02}$ & $-0.85_{-0.24}^{+0.18}$ & $216.51 / 432$ \\
\hline$n=35 \mathrm{H}(\mathrm{z})$ & $0.28_{-0.03}^{+0.02}$ & $-1.14_{-0.28}^{+0.19}$ & $291.56 / 592$ \\
\hline$n=36$ all $\mathrm{H}(\mathrm{z})$ & $0.22_{-0.02}^{+0.01}$ & $-0.82_{-0.09}^{+0.09}$ & $312.38 / 627$ \\
\hline
\end{tabular}
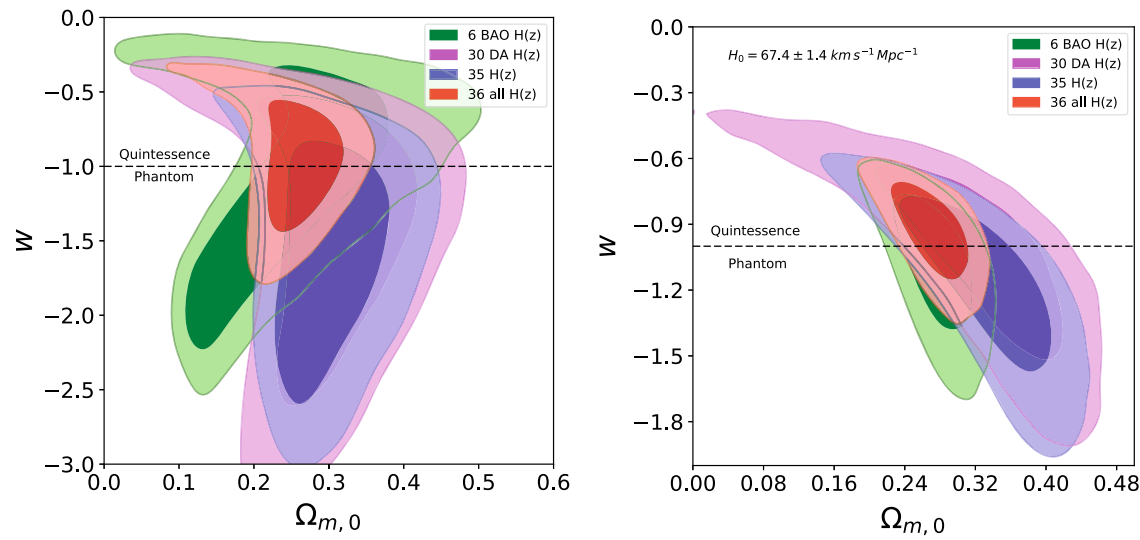

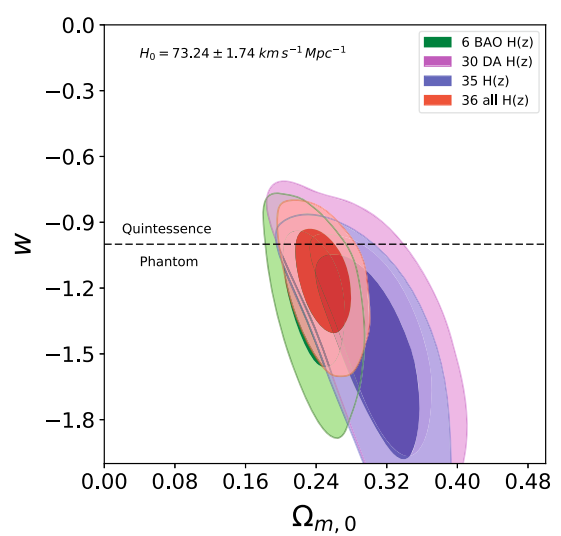

with Gaussian prior $H_{0}=67.4 \pm 1.4 \mathrm{~km} \mathrm{~s}^{-1} \mathrm{Mpc}^{-1}$ from Planck result [20] and lower right one with prior $H_{0}=73.24 \pm 1.74 \mathrm{~km} \mathrm{~s}^{-1} M p c^{-1}$ from local measurements [37]

sitive to the value of $H_{0}$, which is consistent with findings of Farooq [35]. When we use $H(z)$ measurements from BAO and DA techniques separately, the results are different: $H(z)$ behavior $(w<-1)$. The conclusion is that fits are very sen-
$\Lambda \mathrm{CDM}$ where $w=-1$. However, the prior $H_{0}=73.24 \pm$
$1.74 \mathrm{~km} \mathrm{~s}^{-1} \mathrm{Mpc}^{-1}$ from Riess et al. [36], favors Phantom

obtained with the expansion rate measurements $H(z)$. Upper plot was obtained using the reduced Chi-square function Eq. (9). Lower left one 


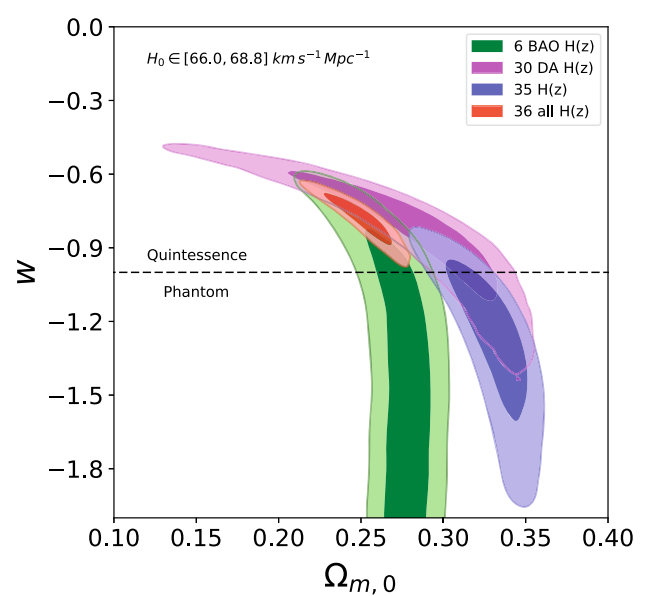

Fig. 3 Constraints on the parameters of wCDM cosmological model obtained using $O m h^{2}\left(z_{i}, z_{j}\right)$ two-point diagnostics. Left panel: with uniform prior $H_{0} \in[66.0,68.8] \mathrm{km} \mathrm{s}^{-1} \mathrm{Mpc}^{-1}$ correspond-

Table 3 Best fitted parameters in CPL cosmological model using $H(z)$ data alone and $O m h^{2}\left(z_{i}, z_{j}\right)$ two-point diagnostics. Fits done on differential ages of cosmic chronometers (DA) and on the full sample

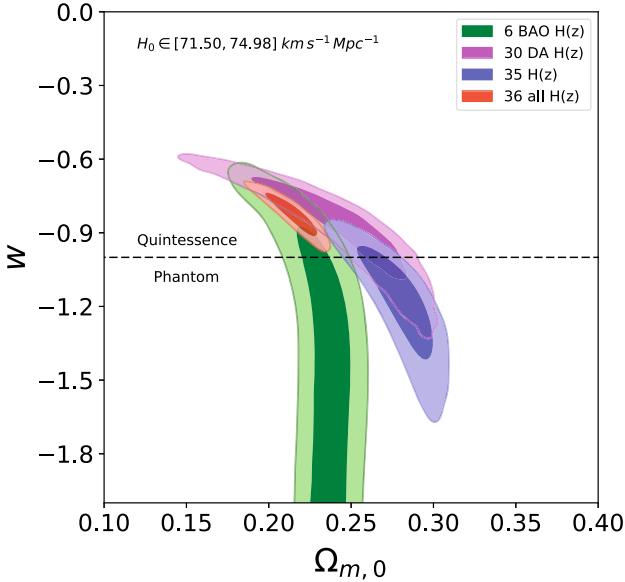

ing to Planck result [20]; Right panel: with uniform prior $H_{0} \in$ [71.50, 74.98] $\mathrm{km} \mathrm{s}^{-1} \mathrm{Mpc}^{-1}$ corresponding to the results from Riess et al. [37]

enriched with BAO data. Priors on $H_{0}$ were taken after Planck [20] and after Riess et al. [37]

\begin{tabular}{|c|c|c|c|c|}
\hline & $\Omega_{m, 0}$ & $w_{0}$ & $w_{a}$ & $\chi_{\text {d.o.f }}^{2}$ \\
\hline \multicolumn{5}{|l|}{$\chi_{H(z)}^{2}(\mathbf{p})$ reduced Chi-square } \\
\hline$n=30 \mathrm{DA} \mathrm{H}(\mathrm{z})$ & $0.40_{-0.19}^{+0.42}$ & $-0.35_{-2.20}^{+5.19}$ & $-0.65_{-35.23}^{+4.11}$ & $14.28 / 27$ \\
\hline$n=36$ all $\mathrm{H}(\mathrm{z})$ & $0.27_{-0.14}^{+0.17}$ & $-0.91_{-0.82}^{+1.14}$ & $0.73_{-4.08}^{+1.75}$ & $17.29 / 33$ \\
\hline $\mathcal{L}_{H}(\mathbf{p})$ Gaussian prior & \multicolumn{4}{|l|}{$H_{0}=67.4 \pm 1.4 \mathrm{~km} \mathrm{~s}^{-1} M p c^{-1}$} \\
\hline$n=30 \mathrm{DA} \mathrm{H}(\mathrm{z})$ & $0.41_{-0.10}^{+0.06}$ & $-0.76_{-0.82}^{+1.17}$ & $0.05_{-15.90}^{+1.93}$ & $15.12 / 27$ \\
\hline$n=36$ all $\mathrm{H}(\mathrm{z})$ & $0.30_{-0.11}^{+0.05}$ & $-0.84_{-0.38}^{+0.35}$ & $0.73_{-3.37}^{+0.66}$ & $18.50 / 33$ \\
\hline $\mathcal{L}_{H}(\mathbf{p})$ Gaussian prior & \multicolumn{4}{|l|}{$H_{0}=73.24 \pm 1.74 \mathrm{~km} \mathrm{~s}^{-1} M p c^{-1}$} \\
\hline$n=30 \mathrm{DA} \mathrm{H}(\mathrm{z})$ & $0.35_{-0.10}^{+0.05}$ & $-1.27_{-0.96}^{+0.91}$ & $1.30_{-13.92}^{+1.99}$ & $15.00 / 27$ \\
\hline$n=36$ all $\mathrm{H}(\mathrm{z})$ & $0.25_{-0.09}^{+0.04}$ & $-1.17_{-0.40}^{+0.30}$ & $1.17_{-2.41}^{+0.71}$ & $18.54 / 33$ \\
\hline$\chi_{O m h^{2}}^{2}\left(H_{0}, \mathbf{p}\right)$ Uniform prior & \multicolumn{4}{|l|}{$H_{0} \in[66.0,68.8] \mathrm{km} \mathrm{s}^{-1} M p c^{-1}$} \\
\hline$n=30 \mathrm{DA} \mathrm{H}(\mathrm{z})$ & $0.29_{-0.16}^{+0.06}$ & $-0.80_{-0.45}^{+0.24}$ & $0.65_{-0.97}^{+0.76}$ & $217.37 / 431$ \\
\hline$n=36$ all $\mathrm{H}(\mathrm{z})$ & $0.20_{-0.11}^{+0.06}$ & $-0.82_{-0.17}^{+0.18}$ & $0.71_{-0.38}^{+0.26}$ & $309.95 / 626$ \\
\hline$\chi_{O m h^{2}}^{2}\left(H_{0}, \mathbf{p}\right)$ Uniform prior & \multicolumn{4}{|l|}{$H_{0} \in[71.50,74.98] \mathrm{km} \mathrm{s}^{-1} M p c^{-1}$} \\
\hline$n=30 \mathrm{DA} \mathrm{H}(\mathrm{z})$ & $0.23_{-0.19}^{+0.05}$ & $-0.87_{-0.33}^{+0.25}$ & $0.74_{-0.78}^{+0.57}$ & $216.96 / 431$ \\
\hline$n=36$ all $\mathrm{H}(\mathrm{z})$ & $0.17_{-0.09}^{+0.04}$ & $-0.88_{-0.15}^{+0.15}$ & $0.79_{-0.34}^{+0.24}$ & $308.82 / 626$ \\
\hline
\end{tabular}

data from DA favor quintessence $(w>-1)$ while $H(z)$ data from BAO favor phantom $(w<-1)$ fields. Moreover the $H(z=2.34)$ point has a big leverage on the final results. This is consistent with conclusions of our previous works [15,21]. The reason lies in different systematic effects between BAO and DA. Better restrictive power of $O m h^{2}\left(z_{i}, z_{j}\right)$ as compared with $H(z)$ technique can be understood in terms of the sample size. Namely, a sample of $n H(z)$ measurements provides us with $\frac{n(n-1)}{2} O m h^{2}\left(z_{i}, z_{j}\right)$ data-points. This advantage does not show up for small samples like $n=6 \mathrm{BAO}$ $H(z)$ data-points. However, the $O \operatorname{Omh}^{2}\left(z_{i}, z_{j}\right)$ diagnostic have a certain drawback: because of $H_{0}$ is strongly degenerated with other cosmological parameters, it should be better to give a prior $H_{0}$ value.

Finally the results concerning CPL parametrization are shown in Table 3 and on Fig. 4 for $30 \mathrm{DA} H(z)$ and the whole $36 H(z)$ sample. The $\Lambda$ CDM model in which $w_{0}=-1$ and $w_{a}=0$ is identified in Fig. 4 by a black star. The $O m h^{2}\left(z_{i}, z_{j}\right)$ diagnostics provides much more stringent results for the dark energy equation of state. For the 30 DA $H(z)$, i.e. the homogeneous sample of cosmic chronometers the black star indicating $\triangle \mathrm{CDM}$ model stays at the edge of 

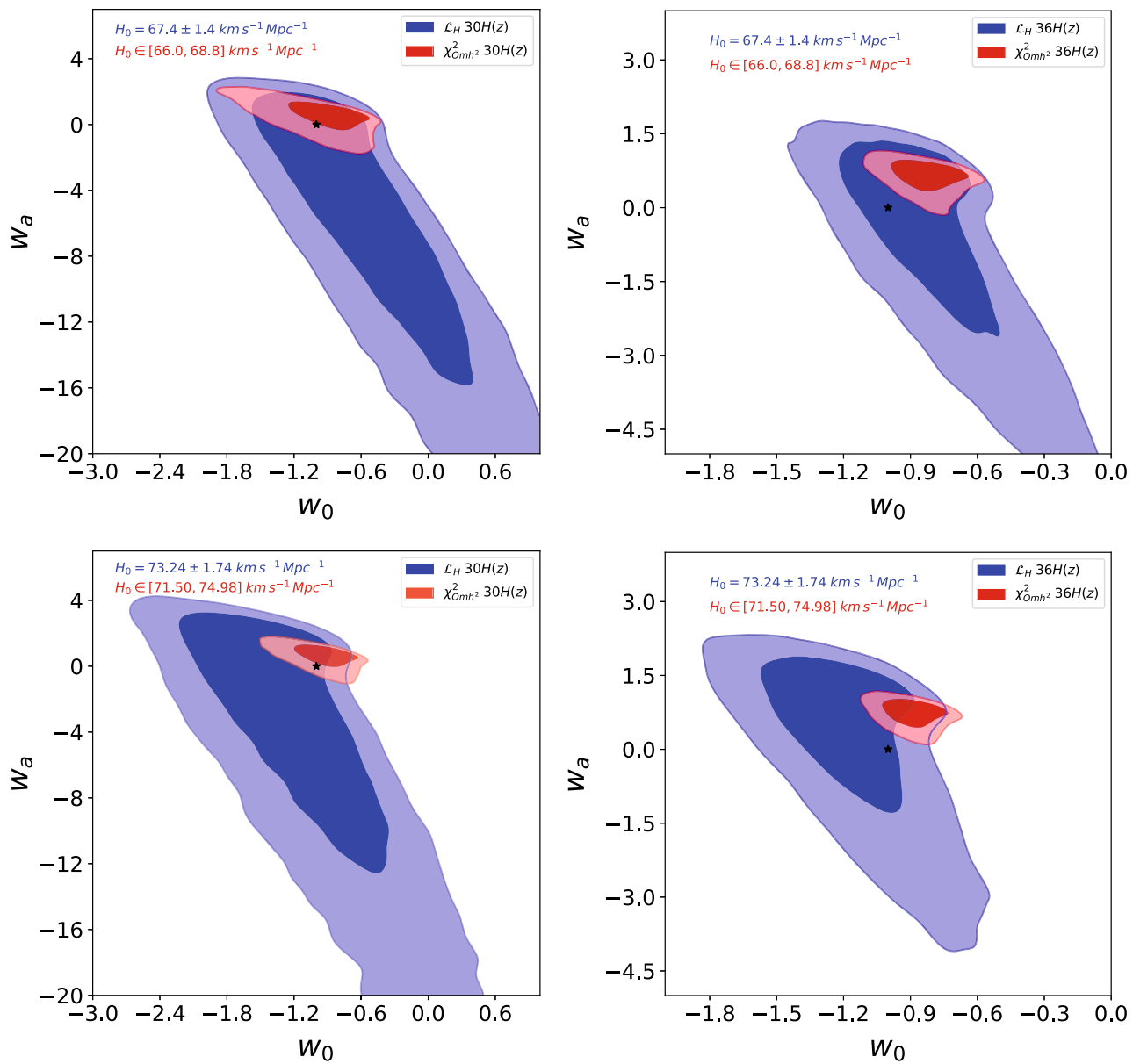

Fig. 4 Constraints on the CPL parameters from 30 DA H(z) data points and 36 total sample. Blue contour: hubble parameters constraint with $H_{0}$ Gaussian priors; Red contour: $O m h^{2}\left(z_{i}, z_{j}\right)$ method constraint with

$H_{0}$ Uniform priors. Left two panels assume $H_{0}$ prior from Planck result [20] and the right two panels correspond to the $H_{0}$ from Riess et al. [37]

$1 \sigma$ confidence region, while it is outside this region for the full, mixed sample of $n=36$ data points. This illustrates the aforementioned systematic effects associated with BAO measurements.

\section{Conclusion}

With increasing number of cosmic chronometers [13,14] covering bigger redshift range, we are starting to directly probe the expansion of the Universe through measurements of its expansion rates $H(z)$ at different epochs. More importantly this sort of measurements is not entangled with cosmic distance ladder considerations or any other calibrations pre-assuming cosmological model. However, there have been some misunderstanding in this respect since additional measurements of $H(z)$ from $\mathrm{BAO}$ peaks location were used in the literature as well. In order to discuss this issue and show

the performance of $H(z)$ data in the context of cosmological model testing, we used recently most complete, mixed data coming from differential ages of passively evolving galaxies together with BAO data-points. Besides such full, inhomogeneous data-set we considered homogeneous sub-samples as well. One of the conclusions was that BAO and DA data should not be mixed together for the purpose of testing cosmological models. This can be understood because BAO technique pre-assumes cosmological model in order to disentangle BAO peak position from the redshift-space distortions due to peculiar velocities of galaxies. Indications of a bias introduced by BAO data has also been noticed in Zheng et al. [21].

In this paper we used both pure expansion rates $H(z)$ and two point diagnostics $\operatorname{Omh}^{2}\left(z_{i}, z_{j}\right)$. The latter has originally been invoked as a litmus test for the $\Lambda \mathrm{CDM}$. There were ideas for using it in broader context [17] illustrated with simulated future data. Here, we applied the two-point diag- 
nostics on the real data and demonstrated that they are able to give much stringent constraints on cosmological parameters. This is because of enhanced size of the data-set: from $n$ original $H(z)$ data-points one can get $n(n-1) / 2$ two-point diagnostics. The price one pays is that they are strongly correlated. Let us stress that the Chi-square function we used was not meant to follow the Chi-square distribution, but it only served a purpose to define the likelihood function to be maximized with MCMC simulations. Even though the constraining power of $\mathrm{Omh}^{2}\left(z_{i}, z_{j}\right)$ two-point diagnostics is considerable, it suffers from being sensitive to the $H_{0}$ prior. Hence the performance of this method crucially depends on our knowledge about the correct value of the Hubble constant. When this work has been completed, Leaf and Melia [38] published an important paper in which they introduced a new type of two-point diagnostics, completely independent on the Hubble constant $H_{0}$. They also gave much more rigorous treatment of statistical properties of this diagnostics. It would be interesting to use their approach in a similar way we did in this paper.

Acknowledgements This work was supported by National Key Research and Development Program of China No. 2017YFA0402600, the National Basic Science Program (Project 973) of China under (Grant No. 2014CB845800), the National Natural Science Foundation of China under Grants Nos. 11503001, 11690023, 11373014, and 11633001, the Strategic Priority Research Program of the Chinese Academy of Sciences, Grant No. XDB23000000, the Interdiscipline Research Funds of Beijing Normal University, and the Opening Project of Key Laboratory of Computational Astrophysics, National Astronomical Observatories, Chinese Academy of Sciences. This research was also partly supported by the Poland-China Scientific \& Technological Cooperation Committee Project No. 35-4. M.B. was supported by Foreign Talent Introducing Project and Special Fund Support of Foreign Knowledge Introducing Project in China.

Open Access This article is distributed under the terms of the Creative Commons Attribution 4.0 International License (http://creativecomm ons.org/licenses/by/4.0/), which permits unrestricted use, distribution, and reproduction in any medium, provided you give appropriate credit to the original author(s) and the source, provide a link to the Creative Commons license, and indicate if changes were made. Funded by SCOAP ${ }^{3}$.
3. T. Clifton, P.G. Ferreira, A. Padilla, C. Skordis, Phys. Rep. 513, 1 (2012)

4. J. Frieman, M. Turner, D. Huterer, Ann. Rev. Astron. Astrophys. 46, 385-432 (2008)

5. T. Buchert, A.A. Coley, H. Kleinert, B.F. Roukema, D.L. Wiltshire, Int. J. Mod. Phys. D 25, 1630007 (2016)

6. M. Chevalier, D. Polarski, Int. J. Mod. Phys. D 10, 213-224 (2001)

7. E.V. Linder, Phys. Rev. Lett. 90, 091301 (2003)

8. S. Cao et al., JCAP 03, 016 (2012)

9. S. Cao et al., PhRvD 90, 083006 (2014)

10. S. Cao et al., ApJ 806, 185 (2015)

11. S. Cao et al., JCAP 02, 012 (2017)

12. S. Cao et al., A\&A 606, A15 (2017)

13. M. Moresco, Mon. Not. R. Astron. Soc. 450, L16 (2015)

14. M. Moresco et al., JCAP 05, 014 (2016)

15. X. Ding, M. Biesiada, S. Cao, Z.X. Li, Z.H. Zhu, Astrophys. J. Lett. 803, L22 (2015)

16. M. Moresco et al., JCAP 12, 029 (2016)

17. V. Sahni, A. Shafieloo, A.A. Starobinsky, Phys. Rev. D 78, 103502 (2008)

18. A. Shafieloo, V. Sahni, A.A. Starobinsky, Phys. Rev. D 86, 103527 (2012)

19. V. Sahni, A. Shafieloo, A.A. Starobinsky, Astrophys. J. Lett. 793, L40 (2014)

20. P.A.R. Ade et al. [Planck Collaboration], Astron. Astrophys. 571, A16 (2014)

21. X.G. Zheng, X.H. Ding, M. Biesiada, S. Cao, Z.H. Zhu, Astrophys. J. 825, 17 (2016)

22. S. Cao et al., A\&A 529, A61 (2011)

23. S. Cao et al., MNRAS 416, 1099 (2011)

24. S. Cao et al., IJMPD 22, 1350082 (2013)

25. Y. Chen et al., JCAP 02, 010 (2015)

26. R. Jimenez, L. Verde, T. Treu, D. Stern, Astrophys. J. 593, 622 (2003)

27. C. Zhang et al., Res. Astron. Astrophys. 14, 1221 (2014)

28. M. Moresco et al., JCAP 08, 006 (2012)

29. J. Simon, L. Verde, R. Jimenez, Phys. Rev. D 71, 123001 (2005)

30. D. Stern et al., JCAP 02, 008 (2010)

31. L. Anderson et al., Mon. Not. R. Astron. Soc. 439, 83 (2014)

32. C. Blake et al., Mon. Not. R. Astron. Soc. 425, 405 (2012)

33. C.H. Chuang, Y. Wang, Mon. Not. R. Astron. Soc. 435, 255-262 (2013)

34. T. Delubac et al., Astron. Astrophys. 574, A59 (2015)

35. M.O. Farooq, arXiv:1309.3710

36. A.G. Riess et al., Astrophys. J. 730, 119 (2011)

37. A.G. Riess et al., Astrophys. J. 826, 56 (2016)

38. K. Leaf, F. Melia, Mon. Not. R. Astron. Soc. 470, 2320 (2017)

\section{References}

1. A.G. Riess et al., Astron. J. 116, 1009 (1998)

2. S. Perlmutter et al., Astrophys. J. 517, 565 (1999) 